\title{
APROXIMATION OF FATIGUE CURVE AND FATIGUE LIMIT OF FIBRE COMPOSITE USING RANDOM DANIELS' SEQUENCE AND MARKOV CHAINS
}

\author{
Yuri Paramonov ${ }^{1}$, Vjacheslavs Cimanis ${ }^{2}$, Sergejs Varickis ${ }^{2}$ \\ Riga Technical University, Riga, Latvia \\ E-mails: 1yuri.paramonov@gmail.com (corresponding author); ${ }^{2}$ chiamian@inbox.lv; ${ }^{3}$ serhiomail@inbox.lv
}

Received 22 February 2013; accepted 03 September 2013

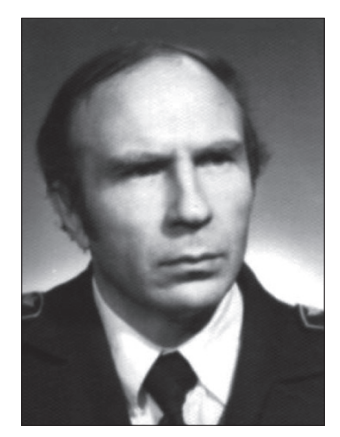

Yuri PARAMONOV. Prof. Dr Habil.

Date and place of birth: 28 March, 1938, Leningrad, Russia.

Education: Riga Aviation Engineering Military High School, Mechanical Engineering Diploma with Gold Medal (1960). Riga Civil Aviation Eng. Institute, Doctor Sc. Eng. Degree (1965).

Latvian Academy of Sciences, High Doctor Degree in Technical Cybernetics (1974). Riga Aviation University and Latvian Academy of Science, Doctor Habilitus Degree in Engineering (1993).

Research interests: reliability of technical systems; mathematical statistics; loads, structure and strength analysis of transport vehicle.

Publications: 228, including ten monographs and textbooks.

Honours, awards: Honoured Scientist of Latvian Soviet Socialist Republic (1983); order: Honour Decoration (1971); medals: For Valiant Labour (1970), Labour Veteran (1985); Nominations for an International Man of the Year for 1997/98 by the International Biographical Centre of Cambridge, and Great Minds of the $21^{\text {st }}$ Century by American Biographical Institute. Present position: Professor in Riga Technical University.

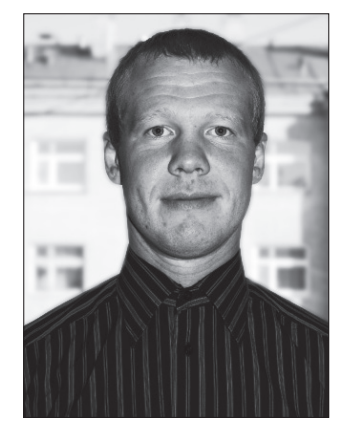

\author{
Vjacheslavs CIMANIS \\ Date and place of birth: 23 April 1986, Riga, Latvia. \\ Education: Riga Technical University, Faculty of Transport and Mechanical Engineering, \\ Master's degree (2011). \\ Research interests: strength analysis of composite materials. \\ Present position: doctoral student of Riga Technical University.
}

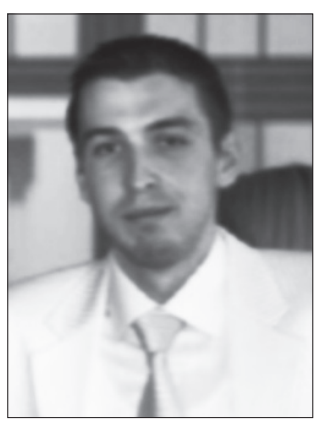

\section{Sergejs VARICKIS}

Date and place of birth: 26 January 1985, Riga, Latvia.

Education: Aviation Institute of Riga Technical University, Bachelor Sc. Eng., engineer's diploma, professionally qualified: Mechanical engineer of aircraft maintenance (2008); Master Sc. Eng.

Degree in aviation (2010), Web technologies school - web programmer (2011).

Research interests: strength analysis of composite materials.

Present position: doctoral student of Riga Technical University.

Abstract. The possibility of using a model based on random Daniels' sequence and Markov chain theory for approximation of S-N fatigue curve of fiber reinforced material is studied. The model allows observing the connection between static strength distribution parameters and parameter of S-N fatigue curve. Although the model is too simple and does not provide numerical correspondence with experimental fatigue test data, it can explain the existence of a fatigue limit and can be used as a nonlinear regression model of S-N fatigue curve when taking into account the 
existence of a random fatigue limit. By using of this model fatigue curve (and especially, fatigue limit) changes as a consequence of tensile strength parameter changes may be predicted. A numerical example of carbon-fiber fatigue test dataset processing is provided.

Keywords: fibers, strength, fatigue, Daniels' sequence, Markov chains.

\section{Introduction}

Each year the use of fiber reinforced material (FRM) in aircraft and other technical structures increases. In order to provide reliability of flight the fatigue phenomenon in this material should be studied. Some research on this topic may already be found (Harris 2003). One of the main quantitative characteristics of this phenomenon is a fatigue curve. Various sources provide their own definition of this concept. One frequently used example is the equation suggested by Weibull: $S-S_{-1}=C(N+B)^{-\alpha}$, where $S_{-1}, C, B$, and $\alpha$ are some parameters, $S$ is the stress amplitude and $N$ is the corresponding average number of cycles. A deep discussion of the considered problem is presented in (Pascual, Meeker 1999). In table 2 of this paper the seven models for estimates of the fatigue curve quantile are given. However, the parameters of these models have no connections with the parameters of the tensile strength distribution of composite material components.

This paper is a review integrating, amending and developing the approach applied in previous works of authors (Paramonov et al. 2006, 2010, 2011, 2012b, c; Cimanis, Paramonov 2012), which are devoted to the connection of tensile strength distribution parameters and parameters of fatigue curve, $\mathrm{S}-\mathrm{N}$, for unidirectional fibre composite using the model, based on the Markov chain (MCh) theory. In (Cimanis, Paramonov 2012; Paramonov et al. 2012b, c) the MCh state space is defined by Daniels' sequence, the definition of which was introduced initially in (Paramonov et al. 2006). It is also connected with the comulative distribution function (cdf) of strength of longitudinal items (LI) (fiber or strands) of unidirectional FRM. Here, the estimate of cdf based on the random sample will be used instead of cdf itself, and the definition of random Daniels' sequence (RDS) is introduced. The "likelihood" of theoretical and experimental fatigue curves may be considered as proof of the model offered in this paper. A numerical example of carbon-fiber fatigue test dataset processing is provided. The paper is an expansion of indeas on the problems considered in book (Paramonov et al. 2011).

The statistical description of the strength of bundles of threads was theoretically described in the papers (Daniels 1945, 1989; Phoenix, Taylor 1973; Smith 1982; Phoenix et al. 1997). Application of the Markov chains (MCh) theory to the analysis of fatigue phenomenon is thoroughly discussed in (Bogdanoff, Kozin 1989; Goda et al. 2006). The result of processing fatigue test dataset using models of fatigue curve with random fatigue limit is presented in paper (Pascual, Meeker 1999).

The model for connection of cdf of tensile strength, fatigue life, residual strength and residual fatigue life (after some preliminary fatigue loading) with the cdf of tensile strength of a composite material component is relatively new. First steps in that direction were made in (Kleinhofs 1983).

In the present article we discuss the probability model of one week micro volumes (WMV) for description of fatigue progress using the definition of the random Daniels' sequence (RDS) and simple Markov chains (MCh), followed by a numerical example of carbon-fiber fatigue test dataset processing.

\section{Model of WMV of unidirectional FRM. The definition of a random Daniels' sequence}

The composite specimen for the test of fatigue life can be regarded as a series of parallel systems (Gucer et al. 1962) every link of which (parallel system) is some WMV, in which gradual accumulation of fatigue damage takes place. After failure of any of these WMVs, the failure of the specimen also takes place. Here we mainly consider the failure of one WMV. This is a special case and the kernel of the whole problem. The use of the considered model of WMV for the analysis of fatigue problem of the specimen as a whole is considered in (Paramonov et al. 2011, 2012a).

First, we assume that the WMV consists of $n_{C}$ LIs in parallel with the applied load shared equally among surviving elements. The value of $n_{C}$ is assumed to be equal to some constant (it is not a random variable). If the sorted strengths of the individual LIs are denoted by $X_{(1)}, X_{(2)}, \ldots, X_{\left(n_{C}\right)}$, the strength of the WMV, $Y$, is provided according to $Y=\max \left\{X_{(k)}\left(n_{C}-k+1\right) / n_{C}: 1 \leq k \leq n_{C}\right\}$. The distribution of $Y$ was investigated by H. E. Daniels (Daniels 1945; Phoenix, Taylor 1973) under the assumption that the fibre strengths, $X_{1}, X_{2}, \ldots, X_{n_{C}}$, are independent random variables with a known common distribution function. R. L. Smith obtains limit theorems as $n_{C} \rightarrow \infty, n_{L} \rightarrow \infty$ simultaneously in consideration of probabilities of large deviations in Daniels' model and improved approximations which lead to significant reductions in error (Smith 1982). If $x_{1: n_{C}}=\left(x_{1}, \ldots, x_{n_{C}}\right)$ is a sample, i.e. a vector of observations of strength of $n_{C}$ LIs of some WMV, $X_{1}, X_{2}, \ldots, X_{n_{C}}$, then while 'developing' the Daniels' model in time, we obtain the following 
sequence, which we refer to as the random Daniels' sequence (RDS), $\left\{s_{0}, s_{1}, s_{2}, \ldots\right\}$ :

$$
s_{i+1}=s /\left(1-\hat{F}_{X}\left(s_{i}\right)\right), \quad i=0,1,2, \ldots, n_{C},
$$

where $s_{0}=s, s$ is the initial nominal stress (initial load of one LI), $\hat{F}_{X}($.$) is the estimate of cdf of strength of a$ LI, which is defined by sample $x_{1: n_{C}}$. In what follows, for definiteness, it is assumed that $s$ is the maximum (nominal) stress of the cycle; items of RDS, $\left\{s_{0}, s_{1}, s_{2}, \ldots\right\}$, are local stresses in a cross section in which some part of LI is destroyed. The following definition of cdf may be used: $\hat{F}_{X}(x)=k(x) / n_{C}$, where $k(x)$ is the number of observations which are lower than or equal to $x$, but here we use the estimate of cdf developed on the base of maximum likelihood estimates of unknown parameters obtained using sample $x_{1: n_{C}}$. The RDS has the following specific features:

1) inequality $\hat{F}_{X}(x) \leq 1$ means that $s_{i+1} \geq s_{i}$;

2) if there is the solution of equation

$$
s=x\left(1-\hat{F}_{X}(x)\right),
$$

then there is some $i^{*}$, such that $s_{i^{*}+1}=s_{i^{*}}$ and the process of increasing $s_{i}$ halts. The maximum value of $s$ which results in the phenomenon is referred to as RDSfatigue-limit (RDSFLm). The solution of the equation (2) exists if $s \leq \max x\left(1-\hat{F}_{X}(x)\right)$. So, for specific $x_{1: n_{C}}$ the specific RDSFLm is equal to $\max x\left(1-\hat{F}_{X}(x)\right)$. But this value is equal to the value of Daniels' tensile strength of bundle of LI. Real fatigue-limit is much lower than the ultimate strength of FRM (USFRM). The decrease of the fatigue limit in comparison with the USFRM may be explained in two ways. Firstly, it may be considered to be the result of some additional stress concentration taking place during repeated load cycles. It may also be maintained that the "local tensile fatigue strength" of LI is lower than the one of single LI. Examples of data processing the fatigue test of carbon-fiber composite using the assumption about development of additional stress concentration for the case $n_{C} \rightarrow \infty$ and $\hat{F}_{X}(x)=F_{X}(x)$ are illustrated in (Cimanis, Paramonov 2012; Paramonov et al. 2012b, c). Here we assume that $n_{C}$ is limited and that the final "local fatigue tensile" strength decreasing in $k_{f}$ time takes place: $X_{L}=X / k_{f}$. In the following we use $F_{X_{L}}($.$) instead of F_{X}($.$) and define the RDSFLm by$ equation $S_{D}=\max x\left(1-\hat{F}_{X_{L}}(x)\right)$. In fact, these two approaches are "convertible" but it appears that the terminology is easyier in this case.

If stress $s$ is more than RDSFLm then items of RDS grow up to infinity. Growth of stress corresponds to the decrease of local specimen cross section area. Let us define that the failure of specimen takes place if local cross section area becomes less than $f_{C}$ (a critical part of still intact LI; initial cross section area is equal to one) of some value. Then critical stress corresponding to this event, $s_{U T}^{*}$, is defined from equation $f_{C}=1-F_{X_{L}}\left(s_{U T}^{*}\right)$ : $s_{U T}^{*}=F_{X_{L}}^{-1}\left(1-f_{C}\right)$. The number $N_{D}=\max \left\{i: s_{i}<s_{U T}^{*}\right\}$ is referred to as Daniels' fatigue life (RDSFLf) at stress $s$. Therefore, there are two different types of RDS: 1 ) RDS is directed upwards to infinity if level of cycling stress $s$ is greater than RDSFLm, in which case RDSFLf is limited; 2) after a slight increase RDS is directed to infinity in a horizontal direction if $s$ is smaller than RDSFLm; in this case RDSFLf is equal to infinity. Examples of processing of fatigue test dataset in which there are right-censored fatigue observations are shown in figures 1 and 2. The dataset employed in this analysis was kindly given to the authors by W. Q. Meeker, who already studied them (Pascual, Meeker 1999) and provides the following description of the data: "the data come from 125 specimens analysed in four-point out-of-plane bending tests of carbon eightharness-satin/epoxy laminate. Both fiber fracture and final specimen fracture occurred simultaneously. Thus, fatigue life is defined as the number of cycles until specimen fracture. The dataset includes 10 right-censored observations (referred to as "runouts" in the fatigue literature)".

The RDSs for different stress levels: $s=340,300$, 280 , and $270 \mathrm{MPa}$ are shown in figure 1a (RDS for $s=$ 380 is not shown because it is too short).

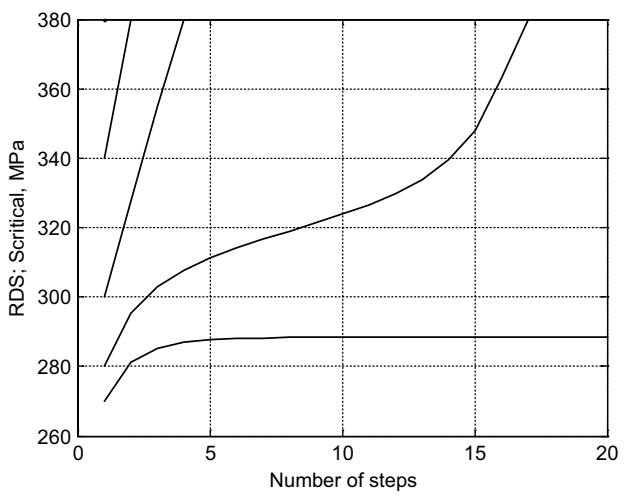

(a)

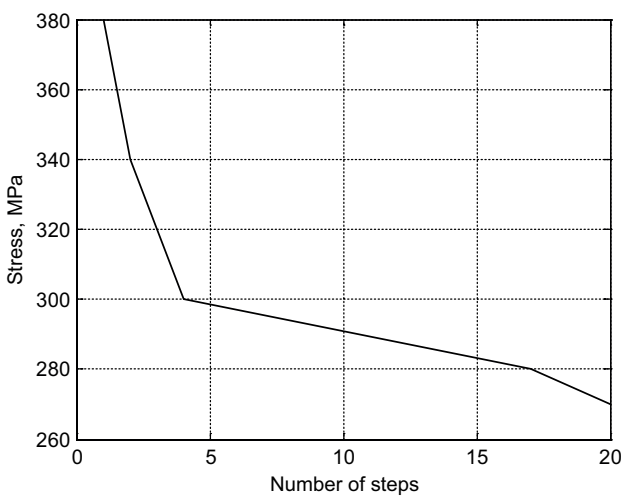

(b)

Fig. 1. Examples of (a) RDS, and (b) fatigue curve $S-N_{D}$ 
Nevertheless, there are some reservations. If $N_{D}=\infty$ then in the figure the "censored" value $N_{D C}=20$ is shown because $\infty$ cannot be shown. Therefore, the output is $N_{D C}=\min \left(N_{D}, 20\right)$.

It is evident that employment of an RDS definition allows to explain the existence of a fatigue limit: for $\mathrm{s}=$ $270 \mathrm{MPa}$ the $N_{D}=\infty$. But the value of $N_{D}$ is very small if $s$ is not very close to the RDSFLm. Therefore, in order to meet the real fatigue life value the value of $N_{D}$ must be "stretched out". This may be achieved by using $k_{D} N_{D}, k_{D} \geq 1$, instead of $N_{D}$. Two other approaches are offered in (Paramonov et al. 2011, 2012b, c; Cimanis, Paramonov 2012). In Paramonov et al. (2011, 2012b), Cimanis and Paramonov (2012) the MCh model was used. Evidently, accumulation of some energy and corresponding number of cycles are needed to cause failure of LIs even if their local strength already is lower than stress. Therefore, in (Paramonov et al. 2012b) the $N_{D}$ was connected with the time needed for accumulation of some energy, which is a part of energy necessary for failure of specimen at a tensile test. It is not clear which approach is better and a special investigation is needed to provide an answer.

In this analysis the first approach and Monte Carlo modelling of sequence $\left\{s_{0}, s_{1} \ldots\right\}$ was used. In this case the description of a random process of accumulation of fatigue damage is defined by:

(1) initial (maximum cycle) stress, $s$;

(2) cdf of strength of LIs, $F_{X_{L}}($.$) , taking into account$ the decrease of local stress by $k_{f}$ time;

(3) sample size, $n_{C}$;

(4) method of estimation of $F_{X_{L}}($.$) using sample,$ $x_{L, 1: n_{C}}$.

The description of a specific realization of this process begins with the recording of a specific sample, $x_{L, 1: n_{C}}$, and with the definition of RDS type. For two different types of RDS we consider MChs with two types of absorbing states (see two following sections). In both cases the state space of MCh is connected with items of RDS. In this paper we consider a simple MCh, in which the transition can be made only in the next seniour state. A more complex two dimensional MCh (when the influence of composite matrix is taken into account) is considered in (Paramonov et al. 2011, 2006), however, for the state space of MCh matrix, which is not connected with the RDS. The main object in this paper is the employment of the RDS for explanation of existence of random RDSFLm and for analysis of fatigue RDSFLf processing result of a specific fatigue test (see numerical example).

\section{Simple MCh model of first type}

First of all we are interested in the cases when RDSFLf is final. Let us denote the pair \{nominal stress, $s$; the sample, $\left.x_{L, 1: n_{C}}\right\}$ with which this event takes place by $\left\{s, x_{L, 1: n_{C}}\right\}^{*}$ and corresponding RDS by $\left\{s_{0}, s_{1}, \ldots, s_{r-1}\right\}^{*}$. We regard the MCh as conditional as long as the RDS of this type takes place and the first $r$ states of corresponding $\mathrm{MCh}$ are related with items of Daniels' sequence, $\left\{s_{0}, s_{1}, \ldots, s_{r-1}\right\}^{\star} ;(r+1)$-th is the absorbing state. $S r$, corresponding to the situation when the RDS-item becomes greater than $s_{U T}^{*}$. We assume for simplicity, that only the transitions to the nearest 'senior' states can take place. The following matrix illustrates transition probabilities:

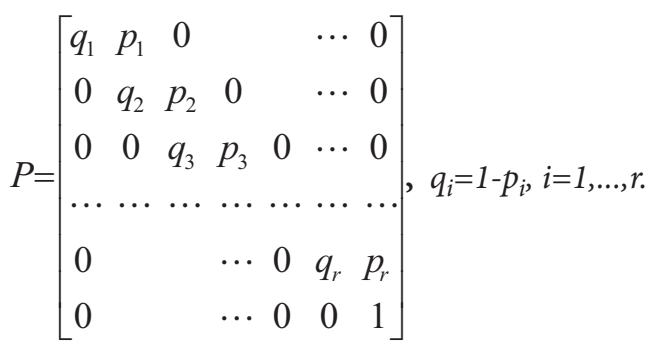

The main characteristics of this type of Markov chain are well known: time until absorption $T_{A}=X_{1}+X_{2}+\ldots+X_{r}$, where $X_{i}$ (time the process spends in $i$-th state) and $i=1, \ldots, r$, are independent random variables which have a geometric distribution with probability mass function $P\left(X_{i}=n\right)=\left(1-p_{i}\right)^{n-1} p_{i}, \quad i=1,2, \ldots \quad$ Expectation value and variance are equal to $E\left(X_{i}\right)=1 / p_{i}$ and $V\left(X_{i}\right)=\left(1-p_{i}\right) / p_{i}^{2}$.

Probability generating function for random variable $T_{A}$ is equal to $G_{T}(z)=\sum_{i=1}^{\infty} p_{T}(i) z^{i}=\prod_{i=1}^{r} \frac{z p_{i}}{1-z\left(1-p_{i}\right)}$, the cdf is equal to $\pi P^{t} b$, where $\pi$ is a row vector of a priori probabilities (in the simplest case $\pi$ is a row vector of the type $(1,0, \ldots, 0))$ and vector column $b$ is vector of the type $(0, \ldots 0,1)$. The new steps which we offer are: 1) the connection of transition probabilities with tensile strength distribution parameter and parameters of cycles of fatigue loading, and 2) the connection of MCh state space with RDS. Let us denote by $\theta$ the vector parameter, the components of which include the parameters of the distribution functions of local strength $F_{X_{L}}($.$) , and some other parameters of the model \left(f_{C}\right.$, $\left.k_{f}, n_{C}, \ldots\right)$. Matrix $P$ and cdf of $T_{A}$ are functions of parameter $\theta$ and the pair $\left\{s, x_{L, 1: n_{C}}\right\}^{*}$ :

$$
F_{T_{A}}\left(t ; \theta,\left\{s, x_{L, 1: n_{C}}\right\}^{*}\right)=\pi P^{t} b, t=1,2,3, \ldots .
$$

It is assumed that one step of Markov chain case corresponds to $k_{M}$ cycles in general ( $k_{M}$ is also a component of vector $\theta$ ). Then the fatigue life (the fatigue cycle number up to specimen failure) $T$ is equal to $k_{m} T_{A}$. The $p$-quantile fatigue curve, which defines the fatigue life $t_{p}(s)$ (the number of cycles corresponding to the probability of failure $p$ under a nominal stress $s$ ), and the corresponding mean fatigue curve are defined by equations: 


$$
\begin{aligned}
& t_{p}(s)=k_{m} F_{T_{A}}^{-1}\left(p ; \theta ;\left(s, x_{L, 1: n_{C}}\right)^{*}\right) ; \\
& E(T(s))=\int_{0}^{\infty} t d F_{T_{A}}\left(t ; s, \theta ; x_{L, 1: n_{C}}^{*}\right) .
\end{aligned}
$$

It should be reminded that the sample $x_{L, 1: n_{C}}$ is random. So the matrix $P$, and cdf of $T_{A}$ are also random. A row vector $\pi$ of a priori probabilities and vector column $b$ of the type $(0, \ldots 0,1)^{\prime}$ are also random because their length is defined by random RDS $\left\{s_{0}, s_{1}, \ldots, s_{r-1}, s_{r}\right\}^{*}$. Therefore, it is necessary to calculate the mean values or to find the corresponding quantiles (at specific argument $s$ ) of these functions under the condition that the pair $\left\{s, x_{L, 1: n_{C}}\right\}^{*}$ takes place. Calculation of the corresponding integrals takes up a lot of time, so it is more convenient to use the Monte Carlo (MC) method. First, a sample $x_{L, 1: n_{C}}$ must be obtained. Then for every specific $s$ (the point of S-N curve) the RDS should be calculated and if it is a first type RDS, functions $F_{T A}(),. t_{p}($.$) and E(T(s))$ for this specific stress level, $s$, should be calculated. Modelling of samples should be repeated long enough to reach the necessary precision. The value of part of the MC trials which provide the pairs of the first type, $\left\{s, x_{L, 1: n_{C}}\right\}^{*}$, is an estimate of the probability that Daniels fatigue limit is lower than stress level $s$. Calculation for different $s$ gives the estimate of cdf of random Daniels' fatigue limit.

\section{Simple Markov chain model of second type}

The second type of the MCh corresponds to the situation when stress $s$ is smaller than RDSFLm and RDSFLf is equal to infinity. This case corresponds to the existence of the solution of equation (2). Assuming that $i^{*}=\min \left(i: s_{i+1}=s_{i} ; i=0,1,2, \ldots\right)$, the states of MCh are connected with the first $i^{*}$ items of RDS: $s_{o}, s_{1}, s_{2}, \ldots, s_{i^{*}}$. The last state is the absorbing one. All previous equations for the number of steps of MCh to absorption can be used again but they are not of great interest for the definition of fatigue life, which is in this case infinite. This type of matrix may be useful for calculation of cdf of fatigue life under program fatigue loading, if the loads are included in the program that the fatigue life is limited. Here we do not consider the analysis of fatigue life under program loading. This problem in relation to different types of state space of MCh is considered in (Paramonov et al. 2011, 2006).

It must be noted once more that all equations related to the calculation of cdf of fatigue life using the $\mathrm{MCh}$ of first type are conditional: under the condition that stress $s$ is greater than RDSFLm. Hence, the main interest is the calculation of the probability of this event. The probability can be calculated using the MC method but an approximate value can be obtained using Daniels' result as well: random variable $S_{D}=\max x\left(1-\hat{F}_{X_{L}}(x)\right)$ has an approximately normal distribution with mean value $\mu_{D}=\max x\left(1-F_{X_{L}}(x)\right)=x^{*}\left(1-F_{X_{L}}\left(x^{*}\right)\right)$ and standard deviation $\sigma_{D}=\left(\mu_{D} x^{*} F_{X_{L}}\left(x^{*}\right) / n_{C}\right)^{1 / 2}$ defined by $F_{X_{L}}$ (.) (Daniels 1945, 1989; Smith 1982).

\section{An example of censored data processing using $k_{f m}$-RDS_MCh model}

The studied model will be referred to as $k_{f m}$-RDS_MCh model. For modelling vector $X_{L, 1: n_{C}}=\left(X_{L 1}, \ldots, X_{L n_{C}}\right)$ the Weibull distribution was used with cdf being $F_{X_{L}}(x)=1-\exp \left(-\exp \left(\left(\log (x)-\theta_{0}\right) / \theta_{1}\right)\right), \quad$ where $\theta_{0}=5.99$ and $\theta_{1}=0.1416$. These parameters correspond to the result of tensile test of carbon fiber strands (Kleinhofs 1983): $E(\log (X))=6.44, \sigma(\log (X))=0.1816$ (pity, there are no data of tensile test results of specific carbon fibers or strands in (Pascual, Meeker 1999)). The first value was corrected in accordance with $k_{f m}$-RDS MCh model: $E\left(\log \left(X_{L}\right)\right)=E(\log (X))-\log \left(k_{f}\right)$. The local strength decreasing coefficient $k_{f}=1.7$ was found by fitting the considered dataset. It was excepted that the fatigue failure of specimen corresponds to the failure of some weak micro volume, which consists of $n_{C}=$ 20 longitudinal items (it is assumed that after their failure the "domino-effect" takes place (Paramonov et al. 2011); but the failure of WMV takes part if its critical part $f_{C}=0.5$ is destroyed. Corresponding critical stress $s_{U T}^{*}$ is equal to 379.7. Here and in the following the the $\mathrm{MPa}$ is used as stress unit. Time scale factor $k_{m}$ (N.B. one step of MCh corresponds to $k_{m}$ fatigue loading cycles) is equal to 57.8. This value was also obtained by fitting the test dataset (Pascual, Meeker 1999). It is maintained that, in accordance with Daniels' theory for Weibull distribution of strength of single LI, the parameters of normal distribution of $S_{D}$ (for strength of bundle LIs) are: $\mu_{D}=\theta_{1}^{\theta_{1}} \exp \left(\theta_{0}-\theta_{1}\right)$ and $\left.\sigma_{D}=\mu_{D}\left(\left(\exp \left(\theta_{1}\right)-1\right) / n_{C}\right)^{1 / 2}\right)$. As for the parameter of cdf $F_{X_{L}}$ (.) of local tensile strength mentioned previously $\mu_{D}$ is equal to 263.2 and standard deviation $\sigma_{D}$ is equal to 22.95. It is very important to know the maximum of stress, which is denoted as $\sigma_{\infty}$, for which RDSFLm is more than this value with high proability $p_{\infty}$. Taking into account the normal distribution of $S_{D}$, the following equation is derived: $s_{\infty}=\mu_{D}-\sigma_{D} \Phi^{-1}\left(p_{\infty}\right)$. For $p_{\infty}=0.99$ we have $s_{\infty}=209.8$. Calculation of random RDSFLm should be made for every pair $\{s$, MC-sample, $\left.\left(x_{L 1}, \ldots, x_{L n_{C}}\right)\right\}$ in order to single out the RDS of the first type. In the example considered the calculation was

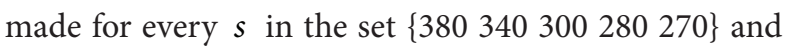
the estimate of cdf of the random RDSFLf and mean conditional fatigue curve $S-N_{M}$ were obtained. This is illustrated in figure 2. The dataset of fatigue test result (Pascual, Meeker 1999) is also shown. 


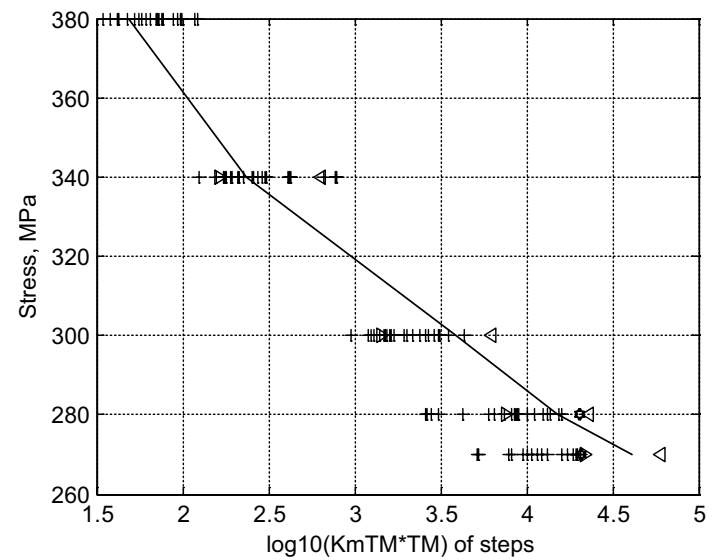

Fig. 2. Mean model fatigue curve (-), two standard deviation intervals $(\bullet, 4)$ for $15 \mathrm{MC}$ trials and test data $(+)$; the censored data are noted by $*$

As mentioned, the processing of the same data was performed in (Pascual, Meeker 1999) using the random fatigue-limit model (RFLM). It must be noted that the random fatigue limit model offered is described in the following way: " $Y$ is the fatigue life and $s$ is the stress level. We model $Y$ as $\log (Y)=\beta_{0}+\beta_{1} \log (S-\gamma)+\varepsilon$, where $\beta_{0}$ and $\beta_{1}$ are fatigue curve coefficients, $\gamma-$ is the fatigue limit of the specimen, $\varepsilon$ is the error term, and $\log$ denotes a natural $\operatorname{logarithm}$. Let $\mathrm{V}=\log (\gamma)$, and supposing that $\mathrm{V}$ has a probability density function (pdf) $f_{V}\left(v ; \mu_{\gamma}, \sigma_{\gamma}\right)=\phi_{V}\left(\left(v-\mu_{\gamma}\right) / \sigma_{\gamma}\right) / \sigma_{\gamma}$ with location and scale parameters $\mu_{\gamma}$ and $\sigma_{\gamma}$, respectively, $\phi_{V}($.$) is either$ the standardised smallest extreme value (sev) or normal pdf. Let $X=\log (\mathrm{s})$ and $W=\log (\mathrm{y})$. Assuming that, conditioned on a fixed value of $V<x, W \mid V$ has a pdf $f_{W \mid V}\left(w ; \beta_{0}, \beta_{1}, \sigma, x, v\right)=(1 / \sigma) \phi_{W \mid V}((w-a) / \sigma) \quad$ with location parameter $a=\beta_{0}+\beta_{1} \log (\exp (x)-\exp (v))$ and scale parameter $\sigma . \phi_{W \mid V}($.$) is either the standardised$ sev or normal pdf. For both random values: $V$ and conditional $W \mid V$, the two mentioned pdfs can be used: for all four versions of sets $\{\mathrm{V}, \mathrm{W} \mid \mathrm{V}\}$ the fitting of the dataset was performed using the RFLM and ML methods.

The model considered in this paper in some way corresponds to the sev distribution of $\mathrm{V}$ and normal distribution of W|V (in accordance with Daniels' proof of assimptotic normal distribution of strength of bundle of fibers). Concerning this case the following estimates are found in (Pascual, Meeker 1999): 5.39 and 0.02 for mean and strandard deviation of V. This corresponds to 219.2 and 133 as estimates of mean and standard deviation of fatigue limit.

In the considered dataset there are 2 and 8 rightcensored data for stress levels 280 and 270 correspondingly (25 fatigue tests for every stress level). This corresponds to estimates of the corresponding probabilities by values 0.08 and 0.32 . In accordance with an assimp- totic normal distribution of RDSFLm with mean 263.2 and standard 22.95 deviation (for this dataset the value $k_{f}=1.7$ was accepted after fitting fatigue life), the probability that RDSFLm is higher than stress levels 280 and 270 is equal to 0.232 and 0.383 correspondingly. The same probability calculated by the MC method is estimated to correspond to values 0.133 and 0.400 .

\section{Conclusion}

The phenomenon of RDS allows to explain the existence of a fatigue limit. The reasonable fitting of fatigue test data of carbon-fiber composite specimen is obtained using a simple Markov chain model with states of space based on random Daniels' sequence assuming the decrease of local tensile strength of LIs in comparison with tensile strength of single LI and some time scale factor. The basic specific feature of models of this type is the possibility of establishing a relation between the parameters of distribution of fatigue life and fatigue limit of a specimen and the parameters of tensile strength of its components. Although the model is too simple and does not provide precise numerical coincidence with experimental fatigue test data, it explains the existence of a fatigue limit and can be used as a nonlinear regression model of an S-N fatigue curve. By using this model, fatigue curve changes as a consequence of tensile strength distribution parameter changes may be predicted.

The great number of unknown parameters of the regression model prevents us (until a successful solution of the problem of creating an effective parameter search algorithm will be found) from recommending it for practical use. However, this model undoubtedly deserves a more extensive and careful verification and has a wider application than only as a source for training courses in higher education institutions.

\section{References}

Bogdanoff, J.; Kozin, F. 1989. Probabilistic Models of Cumulative Damage. Moscow: Mir.

Cimanis, V.; Paramonov, Yu. 2012. Fatigue curve approximation using Daniels' sequence and Markov chains, in $\mathrm{Ma}$ terial Digest of the XXIV Scientific and Practical Conference "Theory and Practice in Physical, Mathematical and Technical Sciences". London: IASHE, 31-33.

Daniels, H. E. 1945. The statistical theory of the strength of bundles of threads, in Proceedings of the Royal Society of London, Series A (183): 405-435.

Daniels, H. E. 1989. The maximum of a Gaussian process whose mean path has a maximum, with an application to the strength of bundles of fibers, Advances in Applied Probability 21(2): 315-333. http://dx.doi.org/10.2307/1427162

Goda, K.; Okabe, T.; Takeda, N. 2006. A strength reliability model of unidirectional fiber-reinforced ceramic matrix composites by Markov process, Advanced Composite Materials 15(3): 263-285.

http://dx.doi.org/10.1163/156855106778392089 
Gucer, D. E.; Gurland, J. 1962. Comparison of the statistics of two fracture modes, Journal of the Mechanics and Physics of Solids 10:365-373. http://dx.doi.org/10.1016/0022-5096(62)90007-8

Harris, B. 2003. Fatigue in Composites. Cambridge, England: Woodhead Publishing Limited. http://dx.doi.org/10.1201/9781439823255

Kleinhofs, M. 1983. Investigation of Static Strength and Fatigue of Composite Material Used in Aircraft Structure: Candidate Degree Thesis, Riga.

Paramonov, Yu.; Andersons, J.; Kleinhofs, M., et al. 2010. MinMaxDM distribution family for analysis of the tensile strength of a unidirectional composite, Mekhanika Kompozitnykh Materialov 46 (3): 397-414.

Paramonov, Yu.; Chatys, R.; Andersons, J., et al. 2012a. Markov model of fatigue of a composite material with poisson process of defect initiation, Mechanics of Composite Materials 48(2): 315-330.

http://dx.doi.org/10.1007/s11029-012-9267-5

Paramonov, Yu.; Chatys, R.; Andersons, J., et al. 2012b. Markov models for tensile and fatigue reliability analysis of unidirectional fiber composite, Reliability \& Risk Analysis: Theory \& Applications 7(3 (26)): 53-65.

Paramonov, Yu.; Cimanis, V.; Kleinhofs, M. 2012c. Studying fatigue curve approximation using Daniels' sequence and Markov chain theory, in Proceeding of the 12th International Conference "Reliability and Statistics in Transportation and Communication - 2012", Riga, 379-383.

Paramonov, Yu.; Kleinhofs, M.; Paramonova, A. 2006. Markov model of connection between the distribution of static strength and fatigue life of a fibrous composite, Mekhanika Kompozitnykh Materialov 42(5): 615-630.

Paramonov, Yu.; Kuznetsov, A.; Kleinhofs, M. 2011. Reliabilty of fatigue-prone airframes and composite materials [online]. Riga: RTU. Available from Internet: http://gnedenko-forum. org/library/Paramonov/Reliability_Paramonov.pdf

Pascual, F. G.; Meeker, W. Q. 1999. Estimating fatigue curves with the random fatigue-limit model, Technometrics 41: 277-302. http://dx.doi.org/10.1080/00401706.1999.10485925

Phoenix, S. L.; Ibnabdeljalil, M.; Hui, C.-Y. 1997. Size effects in the distribution for strength of brittle matrix fibrous composites, International Journal of Solids and Structures 34(5): 545-568. http://dx.doi.org/10.1016/S0020-7683(96)00034-0

Phoenix, S. L.; Taylor, H. M. 1973. The asymptotic strength of a general fiber bundle, Advances in Applied Probability (5): 200-216. http://dx.doi.org/10.2307/1426033

Smith, R. L. 1982. The asymptotic distribution of the strength of a series-parallels system with equal load sharing, $A d v$. in Ann. Prob. (10): 137-171. 